\title{
Subspace and Sparse Reconstruction based Near-field Sources Localization in Uniform Linear Array
}

\author{
Hongqing $\mathrm{Liu}^{1}$, Huan Meng ${ }^{2}$, Lu Gan ${ }^{3}$, Dong $\mathrm{Li}^{4}$, Yi Zhou ${ }^{2}$, and Trieu-Kien \\ Truong ${ }^{5}$ \\ ${ }^{1}$ Chongqing Key Lab of Mobile Communications Technology, Chongqing University of Posts \\ and Telecommunications, Chongqing 400065, China \\ ${ }^{2}$ School of Communication and Information Engineering, Chongqing University of Posts \\ and Telecommunications, Chongqing, China \\ ${ }^{3}$ College of Engineering, Design and Physical Science, Brunel University, London UB8 \\ 3PH, U.K. \\ ${ }^{4}$ Key Laboratory of Aerocraft Tracking Telemetering and Command and Communication, \\ Chongqing University, Chongqing, China \\ ${ }^{5}$ Department of Information Engineering, I-Shou University, Kaohsiung County 84001, \\ Taiwan
}

\begin{abstract}
This work studies the near-field localization problem using a symmetry uniform linear array (ULA). To decouple the range and direction of arrival (DOA), by exploiting the symmetry property of the array, two spatial correlation sequences are constructed, where each sequence only corresponds to one parameter, i.e, DOA or range. After decoupling, an attractive property is that the resulting sequences still share the similar ULA spatial structure. To perform DOA estimation, two approaches have been developed. The first one is based on the power of R (POR) method, which obtains the noise subspace without the eigendecomposition and prior information of the number of sources. The second one is developed using atomic norm minimization, which eliminates the off-grid issue. For range estimation, since the constructed sequence that corresponds to the range parameter shares the same spatial structure with the DOA sequence, the developed approaches are readily applied to obtain the range estimates. The proposed approach is also studied under one-bit measurement to show its
\end{abstract}

${ }^{*}$ Corresponding author E-mail:hongqingliu@outlook.com

Preprint submitted to Elsevier

July 27, 2020 
robustness. The numerical studies including simulation and real-world data demonstrate the performance of the proposed method.

Keywords: Near-field localization, power of R, atomic norm, uniform linear array

\section{Introduction}

Source localization is a fundamental problem in array signal processing such as radar, sonar, and microphone, where the array geometry and phase information are explored to obtain position information of targets [1, It is also a

5 building block for source tracking and source separations [2]. In terms of the distance of the source signal to the reference array, the source localization is categorized as far-field and near-field localizations. For far-field localization, the signal wavefront is assumed to be a plane wave, and therefore, only the direction of arrival (DOA) of the source needs to be estimated. For near-field localization, the signal wavefront is considered as a spherical wave, and hence, the position of the source depends on not only the DOA, but also the range of the source of interest 3 .

Over the past several decades, for the far-field source localization, i.e., DOA estimation, the subspace based methods have been the primary choice. Classical algorithms include, multiple signal classification (MUSIC) [4, maximumlikelihood (ML) [5], subspace fitting [6], estimation of signal parameters via rotational invariance techniques (ESPRIT) [7, and Min-Norm [8] have been developed and successfully applied to different applications. The core idea behind those methods is that the signal subspace is orthogonal to the noise subspace, and by that, the DOA information is extracted. More recently compressed sensing (CS) has been applied to DOA estimation as well [9, 10, 11, 12, 13, 14, 15]. The superior performance is reported by exploring CS concept, but for the fixed dictionary methods, the off-grid issue is present. By utilizing atomic norm minimization, this issue is successfully avoided [15, 16].

For the near-field source localization, the problem becomes a two-dimensional 
(2-D) estimation of range and DOA of the source. To perform efficient estimation, Fresnel approximation is usually adopted to simplify the signal model [17]. Based on this, MUSIC [18, ESPRIT[19], and ML[20] are respectively developed for the near-field source localization. However, the multidimensional search or high-order statistics could be problematic. To decouple the range and the DOA, based on the symmetric property of the uniform linear array (ULA), the localization is realized by a two-stage algorithm, namely DOA estimation is first conducted and then followed by the range estimation. By doing so, the computational complexity is reduced[21, 17]. In each stage, the respective method can be utilized to conduct estimation. For example, in [21, the linear prediction is employed, and in [17, the CS idea is utilized. It is of interest to mention that a lot of studies are also devoted to the localization of the mixed far-field and near-field sources [22, 23, 24, but this is not the focus of this work.

In the above discussions, one assumption implicitly made is that the infinite resolution is employed, which means no quantization. The quantized measurements also enables the cost-effective approaches with a reasonable accuracy and high sampling rate. In [25], one-bit DOA estimation is studied and the beamforming based method is developed to obtain the direction information. In [26], the spatial and spectral information are used to perform one-bit DOA estima45 tion, but the off-grid issue degrades the performance. Based on support vector machine (SVM), a gridless one-bit DOA estimation approach is developed to alleviate grid effect [27. To the best of our knowledge, the study of near-field localization using one-bit measurements is still missing. Therefore, it is important to study its effect on the near-field localization performance.

In this work, the symmetric property of a ULA array is also exploited. In doing so, two special spatial correlation sequences are constructed in which each sequence only corresponds to one parameter, DOA or range, and at the same time, the resulting sequences still preserve the spatial structure of a ULA array. Therefore, to obtain the DOA/range estimation, the traditional method MUSIC 55 can be applied and the search in one-dimension (1-D). In the traditional MUSIC method, the noise subspace is needed, which means the number of sources is 
required. To avoid that, subsequently, we propose to utilize the power of $\mathrm{R}$ (POR) method to obtain the noise subspace without the eigendecomposition and knowing the number of sources. The impacts of POR and finite snapshot are also analyzed theoretically. Furthermore, to explore the sparse promotion technique, the atomic norm minimization is utilized to perform DOA/range estimation by which the off-grid problem is avoided. The one-bit quantization measurement is also studied to show the robustness of the proposed method. Using the one-bit measurements, the spatial correlation sequences needed are estimated and then the developed approaches such as POR and gridless method based atomic norm minimization are applied to perform near-field localization. Finally, the experiments of simulated data and real-world data using microphone array are conducted to demonstrate the localization performance of the proposed approach.

The rest of the paper is organized as follows. The problem formulation of the near-field localization is presented in Section 2. Based on the signal model, the algorithm developments based on POR and atomic norm are presented in 3. In Section 4, performance analysis including integer $p$ and finite snapshot in POR method is provided. The simulations for the proposed approach are presented to demonstrate the effectiveness of the proposed approach in Section 5. Finally, this paper concludes with a brief summary in Section 6 .

\section{Problem Formulations}

The array geometry is given in Figure 1, where the uniform linear array (ULA) is symmetric about the origin that is also the phase reference of the array. In the near-field scenario, namely the ranges of sources are in the Fresnel region $\left[0.62\left(\frac{D^{3}}{\lambda}\right)^{\frac{1}{2}}, \frac{2 D^{2}}{\lambda}\right]$, where $D$ and $\lambda$ respectively indicate the aperture of the array and the wavelength, the received signal by the $m$ th sensor at time $t$ is

$$
y_{m}(t)=\sum_{k=1}^{K} s_{k}(t) \exp \left(j \frac{2 \pi}{\lambda}\left(\ell_{m, k}-\ell_{k}\right)\right)+w_{m}(t), m=-\frac{M-1}{2}, \cdots, \frac{M-1}{2}
$$


where $K$ and $M$ respectively indicate the number of narrowband sources and that of sensors, $\lambda$ is the wavelength, $w_{m}(t)$ denotes the additive noise, and $\ell_{m, k}$ is the distance between the $k$ th $(k=1,2, \cdots, K)$ source and the $m$ th $\left(m=-\frac{M-1}{2}, \cdots, \frac{M-1}{2}\right)$ sensor, given by

$$
\ell_{m, k}=\sqrt{\ell_{k}^{2}+m^{2} d^{2}-2 m d \ell_{k} \sin \left(\theta_{k}\right)}
$$

where $\ell_{k}$ is the distance between $k$ th source and the reference sensor, $d$ is the spacing of sensors, and $\theta_{k}$ is the direction of arrival (DOA).

In a matrix form, (1) is rewritten as

$$
\mathbf{y}(t)=\sum_{k=1}^{K} \mathbf{a}_{\theta, r}\left(\theta_{k}, \ell_{k}\right) s_{k}(t)+\mathbf{w}(t), t=1, \cdots, T
$$

so where $\mathbf{y}(t)=\left[y_{-\frac{M-1}{2}}, \cdots, y_{0}, \cdots, y_{\frac{M-1}{2}}\right]^{\mathrm{T}}$ is the measurement at the sensor positions of $\left[p_{-\frac{M-1}{2}}, \cdots, p_{0}, \cdots, p_{\frac{M-1}{2}}\right]^{\mathrm{T}}, \mathbf{a}_{\theta, r}\left(\theta_{k}, \ell_{k}\right)$ is the steering vector with $\theta_{k}$ and $\ell_{k}$ representing the DOA and range of the $k$ th source, and $T$ is the number of snapshots.

Finally, utilizing the array manifold representation of $\mathbf{A}_{\boldsymbol{\theta}, \mathbf{r}}(\boldsymbol{\theta}, \mathbf{r})=\left[\mathbf{a}\left(\theta_{1}, \ell_{1}\right), \cdots, \mathbf{a}\left(\theta_{K}, \ell_{K}\right)\right]$, (3) in a matrix form is

$$
\mathbf{y}(t)=\mathbf{A}_{\boldsymbol{\theta}, \mathbf{r}}(\boldsymbol{\theta}, \mathbf{r}) \mathbf{s}(t)+\mathbf{w}(t),
$$

where $\mathbf{s}(t)=\left[s_{1}(t), \cdots, \mathbf{s}_{K}(t)\right]^{T}$ is the source vector.

The objective of source localization is to determine the DOA $\theta_{k}$ and range $\ell_{k}$. However, due to nonlinear relationship in (2), the Fresnel approximation is usually utilized [28] i.e.,

$$
\ell_{m, k}=\ell_{k}-m d \sin \left(\theta_{k}\right)+m^{2} d^{2} \frac{\cos ^{2}\left(\theta_{k}\right)}{2 \ell_{k}} .
$$




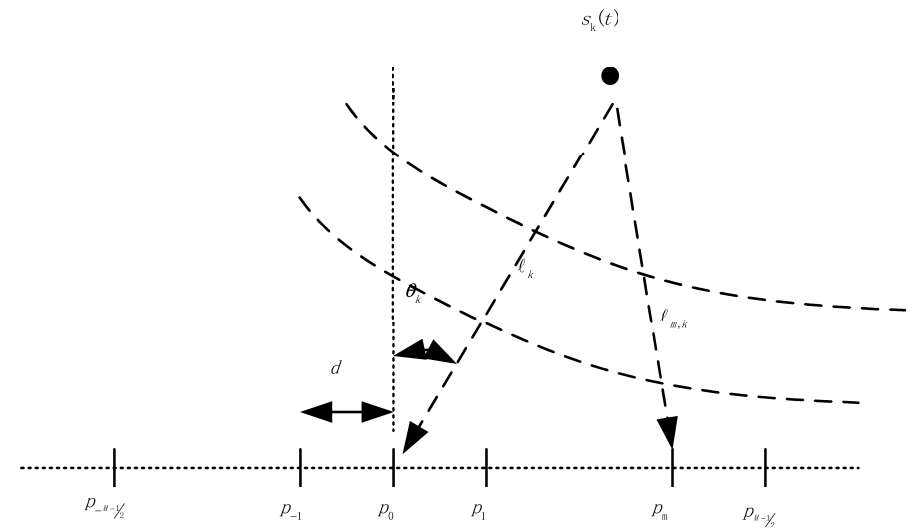

Figure 1: Illustration of array geometry and source placement.

With this approximation, the phase term in (1) is simplified as

$$
\begin{aligned}
\tau_{m, k}=\frac{2 \pi}{\lambda}\left(\ell_{m, k}-\ell_{k}\right) & \approx-m \frac{2 \pi d}{\lambda} \sin \left(\theta_{k}\right)+m^{2} \frac{\pi d^{2}}{\lambda \ell_{k}} \cos ^{2}\left(\theta_{k}\right) \\
& =m \vartheta_{k}+m^{2} \varphi_{k}
\end{aligned}
$$

85 where $\vartheta_{k}=-\frac{2 \pi d}{\lambda} \sin \left(\theta_{k}\right)$ and $\varphi_{k}=\frac{\pi d^{2}}{\lambda \ell_{k}} \cos ^{2}\left(\theta_{k}\right)$.

Substituting (6) into (1), one obtains

$$
y_{m}(t)=\sum_{k=1}^{K} s_{k}(t) \exp \left(j\left(m \vartheta_{k}+m^{2} \varphi_{k}\right)\right)+w_{m}(t) .
$$

Utilizing the matrix notation again, the received array signal is

$$
\mathbf{y}(t)=\mathbf{x}(t)+\mathbf{w}(t)=\mathbf{A}_{\boldsymbol{\vartheta}, \boldsymbol{\varphi}}(\boldsymbol{\vartheta}, \boldsymbol{\varphi}) \mathbf{s}(t)+\mathbf{w}(t)
$$

where $\mathbf{A}_{\vartheta, \varphi}(\boldsymbol{\vartheta}, \boldsymbol{\varphi})=\left[\mathbf{a}\left(\vartheta_{1}, \varphi_{1}\right), \cdots\right.$,

$\left.\mathbf{a}\left(\vartheta_{K}, \varphi_{K}\right)\right]^{\mathrm{T}}$ with $\mathbf{a}_{\vartheta, \varphi}\left(\vartheta_{k}, \varphi_{k}\right)=\left[e^{-j \frac{M-1}{2} \vartheta_{k}} e^{j\left(\frac{M-1}{2}\right)^{2} \varphi_{k}}, \cdots, 1, \cdots, e^{j \frac{M-1}{2} \vartheta_{k}} e^{j\left(\frac{M-1}{2}\right)^{2} \varphi_{k}}\right]^{\mathrm{T}}$, and $\mathbf{s}(t)$ and $\mathbf{w}(t)$ respectively denote the source and noise vectors. It is of interest to point out that based on model of (8), two-dimensional (2D)-MUSIC can be applied directly. However its complexity is high due to the 2D search. 


\section{Algorithm Developments}

\subsection{Signal Conversion}

Prior to developing the algorithm, the following assumptions are made, which are commonly used in practice, and they are

(A1) The sources are mutually independent and independent identically distributed (i.i.d.) complex circular random variables with zero mean and covariance matrix $\mathbf{E}\left(\mathbf{s}(t) \mathbf{s}^{\mathrm{H}}(t)\right)=\operatorname{diag}\left(\sigma_{1}^{2}, \cdots, \sigma_{K}^{2}\right)$, where $\sigma_{k}^{2}$ is the signal power of the $k$ th signal.

(A2) The noise is uncorrelated with the sources and is a spatially white

Gaussian process with zero mean and covariance matrix $\mathbf{E}\left(\mathbf{w}(t) \mathbf{w}^{\mathrm{H}}(t)\right)=\sigma_{w}^{2} \mathbf{I}$, where $\mathbf{I}$ is an identity matrix and $\sigma_{w}^{2}$ is the noise variance.

Utilizing those assumptions, the spatial correlation of the $m$ th and the $n$th sensors are calculated by

$$
\begin{aligned}
r(m, n) & =\mathbf{E}\left(y_{m}(t) y_{n}^{*}(t)\right) \\
& =\sum_{k=1}^{K} \sigma_{k}^{2} \exp \left(j(m-n) \vartheta_{k}+j\left(m^{2}-n^{2}\right) \varphi_{k}\right)+\sigma_{w}^{2} \delta(m-n),
\end{aligned}
$$

where $*$ indicates the conjugate operation and $\delta(\cdot)$ is the Dirac function.

From (9), a more general spatial correlation is defined as

$$
\begin{aligned}
r(\alpha m+\beta, \mu m+\nu) & =\mathbf{E}\left(y_{\alpha m+\beta}(t) y_{\mu m+\nu}^{*}(t)\right) \\
& =\sum_{k=1}^{K} \sigma_{k}^{2} \exp \left(j((\alpha-\mu) m+\beta-\nu) \vartheta_{k}+j\left(\left(\alpha^{2}-\mu^{2}\right) m^{2}+2(\alpha \beta-\mu \nu) m+\left(\beta^{2}-\nu^{2}\right)\right) \varphi_{k}\right) \\
& +\sigma_{w}^{2} \delta((\alpha-\mu) m+\beta-\nu),
\end{aligned}
$$

where $\alpha, \beta, \mu, \nu$ are integers.

Two special correlations are produced from 10 . First, let $\alpha=1, \beta=0, \mu=$ 
$-1, \nu=0$, the following correlation sequence is obtained

$$
r_{1}(m,-m)=\sum_{k=1}^{K} \sigma_{k}^{2} \exp \left(j 2 m \vartheta_{k}\right)+\sigma_{w}^{2} \delta(2 m) .
$$

It is observed that by doing so, the term involving $\varphi_{k}$ is removed in the exponential and only the term of $\vartheta_{k}$ is preserved. By the similar thinking, a second special correlation sequence is constructed that only preserves the term of $\varphi_{k}$. To that end, let $\alpha=\mu=1, \beta=1, \nu=-1$, the second special correlation sequence is produced

$$
r_{2}(m+1, m-1)=\sum_{k=1}^{K} \sigma_{k}^{2} \exp \left(j 2 \vartheta_{k}+j 4 m \varphi_{k}\right) .
$$

Using the matrix representation, (11) is rewritten as

$$
\mathbf{r}_{1}=\mathbf{A}_{\vartheta}(\vartheta) \mathbf{r}_{s}+\sigma_{w}^{2} \mathbf{e}
$$

where $\mathbf{r}_{1}=\left[r(0,0), r(1,-1), \cdots, r\left(\frac{M-1}{2},-\frac{M-1}{2}\right)\right]^{\mathrm{T}}, \mathbf{r}_{s}=\left[\sigma_{1}^{2}, \cdots, \sigma_{K}^{2}\right]^{\mathrm{T}}, \mathbf{e}=$ $105[1,0, \cdots, 0]^{\mathrm{T}}$, and the array manifold $\mathbf{A}_{\vartheta}(\vartheta)=\left[\mathbf{a}_{\vartheta}\left(\vartheta_{1}\right), \cdots, \mathbf{a}_{\vartheta}\left(\vartheta_{K}\right)\right]$ with the steering vector $\mathbf{a}_{\vartheta}\left(\vartheta_{k}\right)=\left[1, e^{j 2 \vartheta_{k}}, \cdots, e^{j 2 \frac{M-1}{2} \vartheta_{k}}\right]^{\mathrm{T}}$.

In a similar fashion, using the matrix form, 12 is rewritten as

$$
\mathbf{r}_{2}=\mathbf{A}_{\varphi}(\varphi) \tilde{\mathbf{r}}_{s}
$$

where $\mathbf{r}_{2}=\left[r(1,-1), r(2,0), r(3,1) \cdots, r\left(\frac{M-1}{2}, \frac{M-1}{2}-2\right)\right]^{\mathrm{T}}, \tilde{\mathbf{r}}_{s}=\left[\sigma_{1}^{2} e^{j 2 \vartheta_{1}}, \cdots, \sigma_{K}^{2} e^{j 2 \vartheta_{K}}\right]^{\mathrm{T}}$ and the array manifold $\mathbf{A}_{\varphi}(\varphi)=\left[\mathbf{a}_{\varphi}\left(\varphi_{1}\right), \cdots, \mathbf{a}_{\varphi}\left(\varphi_{K}\right)\right]$ with the steering vector $\mathbf{a}_{\varphi}\left(\varphi_{k}\right)=\left[1, e^{j 4 \varphi_{k}}, \cdots, e^{j 4\left(\frac{M-1}{2}-1\right) \varphi_{k}}\right]^{\mathrm{T}}$. Let $\alpha_{k}=2 \varphi_{k}$, the steering vector can be reexpressed as $\mathbf{a}_{\alpha}\left(\alpha_{k}\right)=\left[1, e^{j 2 \alpha_{k}}, \cdots, e^{j 2\left(\frac{M-1}{2}-1\right) \alpha_{k}}\right]^{\mathrm{T}}$. With this, 14 becomes

$$
\mathbf{r}_{2}=\mathbf{A}_{\alpha}(\alpha) \tilde{\mathbf{r}}_{s},
$$


It is noted that $\mathbf{A}_{\vartheta}(\vartheta)$ and $\mathbf{A}_{\alpha}(\alpha)$ have the same structure, and therefore, using a new variable $\omega, 13 / 15$ is presented in a unified framework as

$$
\mathbf{r}=\mathbf{A}_{\boldsymbol{\omega}}(\boldsymbol{\omega}) \overline{\mathbf{r}}_{s}+\sigma_{w}^{2} \overline{\mathbf{e}}
$$

where $\mathbf{A}_{\boldsymbol{\omega}}(\boldsymbol{\omega})=\left[\mathbf{a}_{\omega}\left(\omega_{1}\right), \cdots, \mathbf{a}_{\omega}\left(\omega_{K}\right)\right]$, where $\omega$ could be $\vartheta$ in 13 or $\alpha$ in 15. According to equations of 13 and $15, \overline{\mathbf{e}}$ in 16 either $\mathbf{e}$ or a all-zero vector, and correspondingly, $\overline{\mathbf{r}}$ is either $\mathbf{r}_{s}$ or $\tilde{\mathbf{r}}_{s}$. It is also of interest to note that to form the second special correlation, other choices are possible as well 29, 21], for example, the choice of $\alpha=\mu=1, \beta=0, \nu=1$ can also achieve the same objective. To estimate the DOA and range, in this work, the two-stage process is developed where the DOA is estimated based on 13 and range is obtained given (15). Since two models share the same spatial structures, the same procedure can be applied to either sequence to obtain the DOA/range. Therefore, the notation of $\mathbf{r}$ without the subscript is utilized in the following algorithm developments. In what follows, two approaches are developed: the first one is based on the traditional subspace method, i.e., POR algorithm and the other is based on sparse signal reconstruction method, i.e., atomic norm minimization.

\subsection{Proposed Subspace Algorithm}

It is now evident from (16) that the DOA and range estimations can be realized by the well-known MUSIC algorithm. The pseudo covariance matrix, denoted by $\mathbf{R}_{\mathbf{r}}$, is calculated by

$$
\mathbf{R}_{\mathbf{r}}=\mathbf{r r}^{\mathrm{H}}
$$

It is of interest to note that the rank of $\mathbf{R}_{\mathbf{r}}$ equals to the number of sources. Exploiting the eigen value decomposition (EVD), the matrix $\mathbf{R}_{\mathbf{r}}$ is decomposed 
as

$$
\mathbf{R}_{\mathbf{r}}=\left[\begin{array}{ll}
\mathbf{U}_{s} & \mathbf{U}_{n}
\end{array}\right]\left[\begin{array}{cc}
\boldsymbol{\Sigma}_{s}+\sigma_{w}^{2} \mathbf{I} & \mathbf{0} \\
\mathbf{0} & \sigma_{w}^{2} \mathbf{I}
\end{array}\right]\left[\begin{array}{c}
\mathbf{U}_{s}^{\mathrm{H}} \\
\mathbf{U}_{n}^{\mathrm{H}}
\end{array}\right],
$$

where $\mathbf{U}_{s}=\left[u_{1}, \cdots, u_{K}\right]$ and $\mathbf{U}_{n}=\left[u_{K+1}, \cdots, u_{N}\right]$ respectively indicate the signal subspace and noise subspace, and $\boldsymbol{\Sigma}_{s}=\operatorname{diag}\left\{\lambda_{1}^{2}, \cdots, \lambda_{K}^{2}\right\}$. Note that in the case of signal model 16 , since $\mathbf{e}$ has only one nonzero element $\sigma_{w}^{2}$, the eigenvalues of $\mathbf{e e}^{\mathrm{H}}$ has one nonzero component $\sigma_{w}^{4}$. However, in practice, due to limited snapshots, this perfect condition rarely happens. Therefore, the generic model in (18) is still utilized. The effect of limited snapshots will be analyzed by perturbation theory in section 4 .

The subspace theory states that the signal subspace is orthogonal to the noise subspace, and based on this, the estimates of $\vartheta$ and $\alpha$ utilizing one-dimensional (1D)-MUSIC are

$$
\{\hat{\vartheta}, \hat{\alpha}\}=\arg \max _{\omega} \frac{1}{\mathbf{a}_{\omega}^{\mathrm{H}}(\omega) \mathbf{U}_{n} \mathbf{U}_{n}^{\mathrm{H}} \mathbf{a}_{\omega}(\omega)},
$$

where $\mathbf{a}_{\omega}(\omega)=\left[1, e^{j 2 \omega}, \cdots, e^{j 2\left(\frac{M-1}{2}-1\right) \omega}\right]^{\mathrm{T}}$ in which the variable $\omega$ acts the universal surrogate for variables $\vartheta$ and $\alpha$.

The issue of utilizing (12) is to obtain the dimension of the subspace, which can be difficult in practice because the number of sources is required to separate the signal and noise subspaces. To avoid directly employing the EVD, the power of R (POR) technique [30, 31] is utilized. From (18), the following relation holds

$$
\sigma_{w}^{2 p} \mathbf{R}_{\mathbf{r}}^{-p}=\mathbf{U}_{n} \mathbf{U}_{n}^{\mathrm{H}}+\mathbf{U}_{s} \operatorname{diag}\left\{\left(\frac{\sigma_{w}^{2}}{\lambda_{i}^{2}+\sigma_{w}^{2}}\right)^{p}\right\} \mathbf{U}_{s}^{\mathrm{H}},
$$

where $p$ is a positive integer.

From 20, it is obvious that $\left(\frac{\sigma_{w}^{2}}{\lambda_{i}^{2}+\sigma_{w}^{2}}\right)$ is less than one and it approaches zero as $p \rightarrow \infty$. That is,

$$
\lim _{p \rightarrow \infty} \sigma_{w}^{2 p} \mathbf{R}_{\mathbf{r}}^{-p}=\mathbf{U}_{n} \mathbf{U}_{n}^{\mathrm{H}} .
$$


For specific analysis of (21), the more information is provided in Section 4.1,

With this property, the DOA and range estimations can be written without the use of EVD as

$$
\{\hat{\vartheta}, \hat{\alpha}\}=\arg \max _{\omega} \frac{1}{\mathbf{a}_{\omega}^{\mathrm{H}}(\omega) \mathbf{R}_{\mathbf{r}}^{-p} \mathbf{a}_{\omega}(\omega)} .
$$

It is seen that with $(22)$, once the estimations of $\vartheta$ and $\alpha$ are obtained, the final DOA and range values can be simply calculated by

$$
\begin{aligned}
& \hat{\theta}=\arcsin \left(\frac{-\lambda \hat{\vartheta}}{2 \pi d}\right), \\
& \hat{\ell}=\frac{2 \pi d^{2} \cos ^{2}(\hat{\theta})}{\lambda \hat{\alpha}}
\end{aligned}
$$

Clearly, the special spatial correlation sequences are formed at the cost of the array aperture, which in turn may impact the performance of the subspace-based approach. In what follows, the sparsity of the DOA and range are explored, which allows one to achieve super-resolution. In the estimation process, the pairing technique needs to be utilized to determine the correct pair of $\left(\hat{\theta}_{k}, \hat{r}_{k}\right)$, and available methods in [32, 33] can be easily applied to conduct the pairing.

\subsection{Proposed Atomic Minimization}

It is easy to notice that the incoming signals with directions $\left(\theta_{1}, r_{1}\right), \cdots,\left(\theta_{K}, r_{K}\right)$ only occupy certain isolated points in the spatial domain, meaning that the incoming sources are sparse. To exploit that sparsity, the spatial domain can be derived by sampling grid $(\boldsymbol{\theta}, \mathbf{r})$, where $\boldsymbol{\theta}=\left[\theta_{1}, \cdots, \theta_{l}, \cdots, \theta_{J}\right]^{\mathrm{T}}$ and $\mathbf{r}=$ $\left[r_{1}, \cdots, r_{l}, \cdots, r_{J}\right]^{\mathrm{T}}$ with $J$ indicating the number of grids [34]. Using this idea, (16) becomes

$$
\mathbf{r}=\mathbf{A}_{\gamma}(\gamma) \mathbf{u}+\sigma_{w}^{2} \mathbf{e}
$$


By utilizing the sparse property of $\mathbf{u}$, the following optimization is devised

$$
\operatorname{minimize}_{\mathbf{u}}\left\|\mathbf{r}-\mathbf{A}_{\gamma}(\gamma) \mathbf{u}\right\|_{2}^{2}+\lambda\|\mathbf{u}\|_{1},
$$

where $\|\cdot\|_{2}$ is the $\ell_{2}$-norm to measure the data fidelity and $\|\cdot\|_{1}$ is the $\ell_{1}$-norm to promote sparse solution. Once $\mathbf{u}$ is obtained, its nonzero components produce the estimations of the DOA and range.

This so-called grid-based approach inherits a flaw that the sampling grid sometimes does not match the true location of the source, which creates the off-grid problem. When that happens, as expected, the performance of the grid-based one deteriorates significantly. Even though there are some methods developed to migrate this issue, their effects remain to be seen. To address this issue, we untilize, the atomic norm minimization [12, 13, 14, 35]. With that spirit, 16 is rewritten as

$$
\mathbf{r}=\sum_{k=1}^{K} A_{k} \boldsymbol{\nu}_{k}+\sigma_{w}^{2} \mathbf{e}
$$

where $\boldsymbol{\nu}_{k}=\left[1, e^{j \nu_{k}}, e^{j 2 \nu_{k}}, \cdots, e^{j\left(\frac{(M-1)}{2}-1\right) \nu_{k}}\right]^{T}$ with $\nu_{k}=\vartheta_{k}$ or $\nu_{k}=\alpha_{k}$. To estimate $\boldsymbol{\nu}$, based on atomic norm, the following optimization is devised

$$
\operatorname{minimize}_{\mathbf{i}}\|\mathbf{r}-\mathbf{i}\|_{2}^{2}+\tau\|\mathbf{i}\|_{\mathcal{A}}
$$

where $\mathbf{i}=\sum_{k=1}^{K} A_{k} \boldsymbol{\nu}_{k}$ and $\|\cdot\|_{\mathcal{A}}$ is the atomic norm, which is defined by

$$
\|\mathbf{i}\|_{\mathcal{A}}=\inf \left\{\sum_{l} c_{l}: \mathbf{i}=\sum_{l} c_{l} \boldsymbol{\nu}\left(f_{l}\right), c_{l}>0, \boldsymbol{\nu}\left(f_{l}\right) \in \mathcal{A}\right\},
$$

where $\mathcal{A}$ is a collection of atoms and $\boldsymbol{\nu}\left(f_{l}\right)=\left[1, e^{j f_{l}}, e^{j 2 f_{l}}, \cdots, e^{-j\left(\frac{(M-1)}{2}-1\right) f_{l}}\right]^{T}$. To efficiently solve the atomic norm, it can be transformed into the following 
semidefinite programming (SDP) [12]

$$
\begin{aligned}
& \|\mathbf{i}\|_{\mathcal{A}}=\operatorname{minimize}_{t, \mathbf{u}} \frac{1}{2}\left(t+u_{1}\right) \\
& \text { subject to }\left[\begin{array}{cc}
T(\mathbf{u}) & \mathbf{i} \\
\mathbf{i}^{\mathrm{H}} & t
\end{array}\right] \succeq \mathbf{0},
\end{aligned}
$$

where $T(\mathbf{u})$ is an $N \times N$ Toeplitz matrix, given by

$$
T(\mathbf{u})=\left[\begin{array}{cccc}
u_{1} & u_{2} & \cdots & u_{N} \\
u_{2}^{*} & u_{1} & \cdots & u_{N-1} \\
\vdots & \vdots & \vdots & \vdots \\
u_{N}^{*} & u_{N-1}^{*} & \cdots & u_{1}
\end{array}\right]
$$

where $u_{i}$ is the $i$ th component of $\mathbf{u}$.

By utilizing the SDP formulation in 29, the optimization problem 27) can be transformed as

$$
\begin{aligned}
& \operatorname{minimize}_{t, \mathbf{u}, \mathbf{i}} \tau\left(t+u_{1}\right)+\|\mathbf{r}-\mathbf{i}\|_{2}^{2} \\
& \text { subject to }\left[\begin{array}{cc}
T(\mathbf{u}) & \mathbf{i} \\
\mathbf{i}^{\mathrm{H}} & t
\end{array}\right] \succeq \mathbf{0} .
\end{aligned}
$$

To efficiently solve (31), the existing solver of $\mathcal{C} \mathcal{V X}[36$ is utilized to produce the solutions.

\subsection{One-bit DOA and range estimation}

From the received signal in (7), its one-bit quantization is given by

$$
y_{m}^{c}(t)=\frac{1}{\sqrt{2}} \operatorname{csgn}\left\{y_{m}(t)\right\}=\frac{1}{\sqrt{2}}\left\{\operatorname{sgn}\left(\operatorname{Re}\left(y_{m}(t)\right)\right)+j \operatorname{sgn}\left(\operatorname{Im}\left(y_{m}(t)\right)\right)\right\}
$$


where $\operatorname{Re}(\cdot)$ and $\operatorname{Im}(\cdot)$ take the real and imaginary parts of $y_{m}(t)$ and $\operatorname{sgn}(\cdot)$ is a sign function, given by

$$
\operatorname{sgn}(t)=\left\{\begin{array}{cc}
1, & \text { if } t \geq 0 \\
-1, & \text { if } t<0
\end{array}\right.
$$

By arcsine law [37, the auto-correlation of $y_{m}^{c}(t)$, denoted by $r^{c}(m, n)=$ $\mathbf{E}\left(y_{m}^{c}(t) y_{n}^{c *}(t)\right)$, is related to $r(m, n)$ by the following equality

$$
r^{c}(m, n)=\frac{2}{\pi}\{\arcsin \{\operatorname{Re}(r(m, n))\}+j \arcsin \{\operatorname{Im}(r(m, n))\}\} .
$$

According to polarity coincidence correlator (PCC) 38, one obtains

$$
r(m, n)=\sin \left\{\frac{\pi}{2} \operatorname{Re}\left(r^{c}(m, n)\right)\right\}+j \sin \left\{\frac{\pi}{2} \operatorname{Im}\left(r^{c}(m, n)\right)\right\}
$$

In a vector form, the reconstructed correlation sequence is

$$
\hat{\mathbf{r}}=\sin \left\{\frac{\pi}{2} \operatorname{Re}\left(\mathbf{r}^{c}\right)\right\}+j \sin \left\{\frac{\pi}{2} \operatorname{Im}\left(\mathbf{r}^{c}\right)\right\},
$$

where $\mathbf{r}^{c}$ is the spatial correlation calculated from one-bit measurement $y_{m}^{c}(t)$ using different array sensors.

It has been shown that $\mathbf{r}^{c}$ is the best estimate of the original correlation $\mathbf{r}$ [25]. After the corresponding $\hat{\mathbf{r}}$ is recovered using one-bit measurements, the proposed algorithms are ready to apply to perform estimations.

\section{Performance Analysis}

In this section, the POR based approach including the effects of parameter $p$ and finite snapshots is analyzed.

\subsection{Effect of parameter $p$}

160

Lemma 1: When $p \rightarrow \infty$, equation (21) holds, which indicates that the POR approach attains the MUSIC performance. 
Proof: The similar procedure in [30] is utilized. From the EVD, the inverse of $\mathbf{R}_{\mathbf{r}}$ is

$$
\mathbf{R}_{\mathbf{r}}^{-1}=\frac{1}{\sigma_{w}^{2}} \mathbf{U}_{n} \mathbf{U}_{n}^{\mathrm{H}}+\mathbf{U}_{s} \operatorname{diag}\left\{\frac{1}{\lambda_{i}^{2}+\sigma_{w}^{2}}\right\} \mathbf{U}_{s}^{\mathrm{H}} .
$$

Based on (36), one obtains

$$
\mathbf{R}_{\mathbf{r}}^{-p}=\frac{1}{\sigma_{w}^{2 p}} \mathbf{U}_{n} \mathbf{U}_{n}^{\mathrm{H}}+\mathbf{U}_{s} \operatorname{diag}\left\{\frac{1}{\left(\lambda_{i}^{2}+\sigma_{w}^{2}\right)^{p}}\right\} \mathbf{U}_{s}^{\mathrm{H}} .
$$

From Taylor expansion, the term of $\frac{1}{\left(\lambda_{i}^{2}+\sigma_{w}^{2}\right)^{p}}$ is approximated by

$$
\frac{1}{\left(\lambda_{i}^{2}+\sigma_{w}^{2}\right)^{p}} \approx \frac{1}{\lambda_{i}^{2 p}}-\frac{p \sigma_{w}^{2}}{\lambda_{i}^{2(p+1)}}
$$

Substituting (38) into 37$)$ produces

$$
\sigma_{w}^{2 p} \mathbf{R}_{\mathbf{r}}^{-p}=\mathbf{U}_{n} \mathbf{U}_{n}^{\mathrm{H}}+\mathbf{U}_{s} \boldsymbol{\Sigma}_{s}^{-p} \mathbf{U}_{s}^{\mathrm{H}}+\sigma_{w}^{2(p+1)} p \mathbf{U}_{s} \boldsymbol{\Sigma}_{s}^{-(p+1)} \mathbf{U}_{s}^{\mathrm{H}} .
$$

It is obvious that when $p \rightarrow \infty$, equation 21 follows. In that case, the MUSIC performance is attained. However, when $p$ is finite, from $[39$, the energy from signal subspace will leak into the noise subspace, and when that happens, the performance of POR deteriorates, see simulation section for more insights. In the case of $p=1$, this corresponds the Capon beamforming.

The equation 19 is equivalent to

$$
\hat{\omega}=\arg \min _{\omega} f(\omega)
$$

where $f(\omega)=\mathbf{a}_{\omega}^{\mathrm{H}}(\omega) \mathbf{U}_{n} \mathbf{U}_{n}^{\mathrm{H}} \mathbf{a}_{\omega}(\omega)$. By a use of the result in 44, the covariance matrix of estimation errors $\{\hat{\omega}-\omega\}$ using MUSIC algorithm is

$$
\mathbf{C}_{\text {MUSIC }}=\frac{2 \sigma_{w}}{M-1}(\mathbf{H} \odot \mathbf{I})^{-1} \operatorname{Re}\left\{\mathbf{H} \odot\left(\mathbf{A}_{\omega} \mathbf{U} \mathbf{A}_{\omega}\right)^{T}\right\}(\mathbf{H} \odot \mathbf{I})^{-1}
$$


where $\odot$ denotes the element-wise product and $\mathbf{H}=\mathbf{D U}_{n} \mathbf{U}_{n}^{\mathrm{H}} \mathbf{D}$ in which $\mathbf{D}$ is the first derivative of steering vector. In (41), $\mathbf{U}$ is given by

$$
\mathbf{A}_{\omega} \mathbf{U} \mathbf{A}_{\omega}=\mathbf{P}^{-1}+\sigma_{w} \mathbf{P}^{-1}\left(\mathbf{A}_{\omega} \mathbf{A}_{\omega}\right)^{-1} \mathbf{P}^{-1}
$$

where $\mathbf{P}$ is the covariance matrix of $\overline{\mathbf{r}}_{s}$.

Following the similar procedure, the covariance matrix of estimation error using POR based approach is

$$
\mathbf{C}_{\mathrm{POR}}=\frac{2 \sigma_{w}}{M-1}(\overline{\mathbf{H}} \odot \mathbf{I})^{-1} \operatorname{Re}\left\{\tilde{\mathbf{H}} \odot\left(\mathbf{A}_{\omega} \mathbf{U} \mathbf{A}_{\omega}\right)^{T}\right\}(\overline{\mathbf{H}} \odot \mathbf{I})^{-1}
$$

where $\overline{\mathbf{H}}=\mathbf{D R}_{\mathbf{r}}^{-p} \mathbf{D}$ and $\tilde{\mathbf{H}}=\mathbf{D R}_{\mathbf{r}}^{-2 p} \mathbf{D}$.

It is seen that when $p$ is finite, $\mathbf{C}_{\mathrm{POR}} \succeq \mathbf{C}_{\text {MUSIC }}$, which means that the performance of the proposed POR is inferior to that of MUSIC. However, the performance gap vanishes as $p$ grows, as shown in the simulations.

\subsection{Effect of limited snapshots}

In reality, the special correlation sequences are calculated using a finite number of snapshots, which means that the covariance matrix $\mathbf{R}_{\mathbf{r}}$ deviates from true value. By using the perturbation analysis, the effect of finite snapshots are studied. The deviation is denoted by $\Delta \mathbf{R}_{\mathbf{r}}$, and the perturbed covariance matrix is $\mathbf{R}_{r}+\Delta \mathbf{R}_{\mathbf{r}}$. With this, 22 is rewritten as

$$
\hat{\omega}=\arg \min _{\omega} \quad \mathbf{a}_{\omega}^{\mathrm{H}}(\omega)\left(\mathbf{R}_{\mathbf{r}}+\Delta \mathbf{R}_{\mathbf{r}}\right)^{-p} \mathbf{a}_{\omega}(\omega) .
$$

Applying Taylor expansion, $\left(\mathbf{R}_{\mathbf{r}}+\Delta \mathbf{R}_{\mathbf{r}}\right)^{-1}$ is approximated up to the first order by

$$
\left(\mathbf{R}_{\mathbf{r}}+\Delta \mathbf{R}_{\mathbf{r}}\right)^{-1} \approx \mathbf{R}_{\mathbf{r}}^{-1}-\mathbf{R}_{\mathbf{r}}^{-1} \Delta \mathbf{R}_{\mathbf{r}} \mathbf{R}_{\mathbf{r}}^{-1}
$$


Based on 45, $\left(\mathbf{R}_{\mathbf{r}}+\Delta \mathbf{R}_{\mathbf{r}}\right)^{-p}$ is

$$
\left(\mathbf{R}_{\mathbf{r}}+\Delta \mathbf{R}_{\mathbf{r}}\right)^{-p} \approx \mathbf{R}_{\mathbf{r}}^{-p}-\sum_{k=1}^{p} \mathbf{R}_{\mathbf{r}}^{-k} \Delta \mathbf{R}_{\mathbf{r}} \mathbf{R}_{\mathbf{r}}^{(-p+1-k)}
$$

Substituting (46) into (44), one obtains

$$
\begin{aligned}
& \hat{\omega}=\arg \min _{\omega} \quad \mathbf{a}_{\omega}^{\mathrm{H}}(\omega)\left(\mathbf{R}_{\mathbf{r}}^{-p}-\sum_{k=1}^{p} \mathbf{R}_{\mathbf{r}}^{-k} \Delta \mathbf{R}_{\mathbf{r}} \mathbf{R}_{\mathbf{r}}^{(-p+1-k)}\right) \mathbf{a}_{\omega}(\omega) \\
& =\arg \min _{\omega} \quad \mathbf{a}_{\omega}^{\mathrm{H}}(\omega) \mathbf{R}_{\mathbf{r}}^{-p} \mathbf{a}_{\omega}(\omega)-\mathbf{a}_{\omega}^{\mathrm{H}}(\omega)\left(\sum_{k=1}^{p} \mathbf{R}_{\mathbf{r}}^{-k} \Delta \mathbf{R}_{\mathbf{r}} \mathbf{R}_{\mathbf{r}}^{(-p+1-k)}\right) \mathbf{a}_{\omega}(\omega) .
\end{aligned}
$$

The first term in (47) is the POR, which attains the MUSIC performance when $p \rightarrow \infty$. However, the existence of the second term degrades the performance. Consider a special case, where $\Delta \mathbf{R}_{\mathbf{r}}=\mathbf{I}$ and $p=\infty$, and we only take the first component in $\left(\sum_{k=1}^{p} \mathbf{R}_{\mathbf{r}}^{-k} \Delta \mathbf{R}_{\mathbf{r}} \mathbf{R}_{\mathbf{r}}^{(-p+1-k)}\right)$ into calculation. After some algebra, 47 becomes

$$
\hat{\omega}=\arg \min _{\omega} \quad-\mathbf{a}_{\omega}^{\mathrm{H}}(\omega) \mathbf{U}_{s} \operatorname{diag}\left\{\frac{1}{\lambda_{i}^{2}+\sigma_{w}^{2}}\right\} \mathbf{U}_{s}^{\mathrm{H}} \mathbf{a}_{\omega}(\omega) .
$$

It is obvious that using [48] will not produce the correct estimates.

In the case of limited snapshot, the covariance matrix $\mathbf{C}_{\mathrm{POR}}$ becomes

$$
\mathbf{C}_{\mathrm{POR}}=\frac{2 \sigma_{w}}{M-1}(\overline{\mathbf{H}} \odot \mathbf{I})^{-1} \operatorname{Re}\left\{\tilde{\mathbf{H}} \odot\left(\mathbf{A}_{\omega} \mathbf{U} \mathbf{A}_{\omega}\right)^{T}\right\}(\overline{\mathbf{H}} \odot \mathbf{I})^{-1}
$$

where $\overline{\overline{\mathbf{H}}}=\mathbf{D}\left(\mathbf{R}_{\mathbf{r}}+\Delta \mathbf{R}_{\mathbf{r}}\right)^{-p} \mathbf{D}$ and $\tilde{\tilde{\mathbf{H}}}=\mathbf{D}\left(\mathbf{R}_{\mathbf{r}}+\Delta \mathbf{R}_{\mathbf{r}}\right)^{-2 p} \mathbf{D}$.

It is obvious that when the snapshot is limited, $\mathbf{C}_{\mathrm{POR}} \succeq \mathbf{C}_{\text {MUSIC }}$. As the number of snapshot approaches infinity, i.e., $N \rightarrow \infty$, the performance of the POR based method is asymptotically close to the MUSIC.

\subsection{Complexity analysis}

In this subsection, we compare the complexities of the proposed POR and atomic norm based algorithms with that of the MUSIC algorithm and Grid 
matching algorithms. For 1D-MUSIC, in the angle dimension, it involves the EVD decomposition of $\mathbf{R}_{\mathbf{r}}$ of size $(M+1) \times(M+1)$ and requires $\mathcal{O}\left\{(M+1)^{3}\right\}$ operations. After that, for the peak search, its complexity is $\mathcal{O}\left\{N_{\theta}(M+1)(M+\right.$ $1-K)\}$, where $N_{\theta}$ represents the angle grid search. In the range dimension, the EVD takes $\mathcal{O}\left\{M^{3}\right\}$ and the peak search needs $\mathcal{O}\left\{N_{r} M(M-K)\right\}$, where $N_{\mathbf{r}}$ represents the range grid search. Therefore, the total complexity of 1D-MUSIC is

$$
\begin{aligned}
\overline{\mathbf{C}}_{\text {MUSIC }} & =\mathcal{O}\left\{(M+1)^{3}+N_{\theta}(M+1)(M+1-K)+M^{3}+N_{r} M(M-K)\right\} \\
& \approx \mathcal{O}\left\{2 M^{3}+N_{\theta} M^{2}+N_{r} M^{2}\right\} .
\end{aligned}
$$

For the POR method, it is very much the same to the MUSIC, and it is

$$
\overline{\mathbf{C}}_{\mathrm{POR}}=\mathcal{O}\left\{2 M^{3}+N_{\theta} M^{2}+N_{r} M^{2}\right\}
$$

In the sparsely reconstructed grid matching algorithm, the computational complexity of the algorithm consists of two parts. First, the angle grid is constructed, and its computational complexity is $O\left\{(M+1)^{2} N_{\theta}\right\}$. Second, the CVX toolkit is used to produce the solution, and the complexity of the process is $O\left\{2 N_{\theta}^{2}\right\}$. The range dimension can be conducted similarly. The total computational complexity of the grid matching algorithm is

$$
\overline{\mathbf{C}}_{\text {Grid matching }}=O\left\{(M+1)^{2} N_{\theta}+2 N_{\theta}^{2}+N_{r} M^{2}+2 N_{r}^{2}\right\} .
$$

The proposed atomic norm algorithm is to solve the problem of weighted atomic norm minimization, and its complexity is

$$
\overline{\mathbf{C}}_{\text {Atomic norm }}=O\left\{i(M+1)^{3}+i M^{3}\right\},
$$

where $i$ represents the number of iterations. In Table 1, the complexities of each algorithm are summarized, and in Table 2, the running times of different 
Table 1: Complexities of Algorithms

\begin{tabular}{c|c}
\hline MUSIC & $\mathcal{O}\left\{2 M^{3}+N_{\theta} M^{2}+N_{r} M^{2}\right\}$ \\
\hline POR & $\mathcal{O}\left\{2 M^{3}+N_{\theta} M^{2}+N_{r} M^{2}\right\}$ \\
\hline Grid matching & $\mathcal{O}\left\{(M+1)^{2} N_{\theta}+2 N_{\theta}^{2}+N_{r} M^{2}+2 N_{r}^{2}\right\}$ \\
\hline Atomic norm & $\mathcal{O}\left\{i(M+1)^{3}+i M^{3}\right\}$ \\
\hline
\end{tabular}

methods versus number of microphones are also provided.

\section{Numerical Results}

\subsection{Simulations}

In this section, the simulated data are used to demonstrate the effectiveness and accuracy of the proposed algorithm and comparisons with other methods are also provided. To objectively evaluate the system performance, mean square error (MSE) is used. In the experiments, the wavelength is $\lambda=1$, the spacing between the sensors is $d=\frac{\lambda}{4}$, and the number of sensors is $M=15$.

First, the comparisons between POR and MUSIC approaches are conducted. For this comparison, two narrow-band near-field source signals with their positions at $\left(10^{\circ}, 2 \lambda\right)$ and $\left(60^{\circ}, 12 \lambda\right)$ are generated. The estimated results obtained by MUSIC, POR $(p=1)$, Capon, and ESPRIT algorithms are depicted in Fig95 ure 2. In order to clearly observe the estimation results, estimated results of 10 independent runs from two subspace approaches are provided. From figures, the classical MUSIC algorithm in Figure 2a and POR algorithm in Figure 2b, it is seen that both of them are able to obtain the accurate positions of true sources. But unlike MUSIC method POR approach does not require the number of sources and EVD decomposition.

To further demonstrate the performances of the POR method, in Figure 3. the MSEs of the DOA and range obtained by POR method with different values of $p$ versus SNR are provided. In this case, two scenarios of full-bit and one-bit measurements are presented. It is expected, performance increases as SNR increases and they all approach the theoretical bound $C_{\text {theoretical }}$ that is 
calculated by (43). It is observed that $p$ does not play a deciding role as the POR method with different values of $p$ presents a comparable performance, except when SNR is low. Therefore, in the following experiments, the POR method with $p=1$ is utilized, unless stated otherwise. It is also of interest to point out that with one-bit measurement, the corresponding spatial correlation sequence is reconstructed by (35) first, and estimation is carried out by POR method and the theoretical bound is calculated. With less information, one-bit to be exact, the performance degradation is noticed, but its estimation still attains the theoretical bound.

In Figure 4, the MSEs of different approaches are compared against snapshots in the case of full-bit and one-bit measurements. From the figure, the Grid-off it the worst due to the presence of the off-gird. The MSEs of MUSIC and POR methods overlap, which agrees with the theoretical analysis when the snapshot is moderate. With one-bit measurement, the Grid matching approach achieves a perfect estimate when the snapshot exceeds 250 in terms of DOA estimation, demonstrated in Figure 4a, The atomic norm based method offers a promising performance in both scenarios.

Finally, in Figure 5, the MSEs of different approaches are compared in the case of full-bit and one-bit measurements. From the figure, the Grid-off performs 225 the worst because of the presence of the off-gird, which is also consistent with the conclusion in Figure 4. The MSEs of MUSIC and POR methods overlap, suggesting they produce the same performance, which agrees with the theoretical analysis when the SNR/snapshot is moderate, they present the similar performances. With full-bit measurement, the Grid matching approach achieves a perfect estimate when the SNR exceeds $10 \mathrm{~dB}$, demonstrated in Figure 5a With one-bit quantization, however, the Grid matching approach deteriorates. The atomic norm based method offers a promising performance in both scenarios and it approaches the CRB. The running times of different methods are provided in Table 2, using a Intel Dual Core i3 with CPU $2.4 \mathrm{GHz}$ and MAT-

235 LAB. The results show that the MUSIC and POR execute similarly in time. In a comparison of sparse reconstruction based method, the atomic norm based 


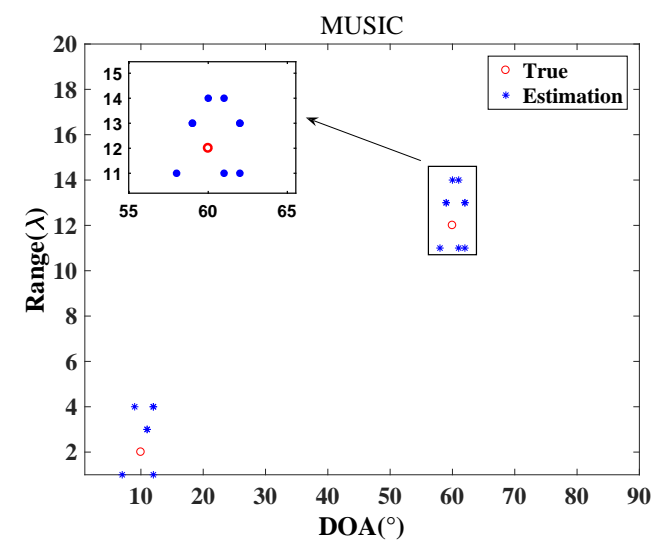

(a)

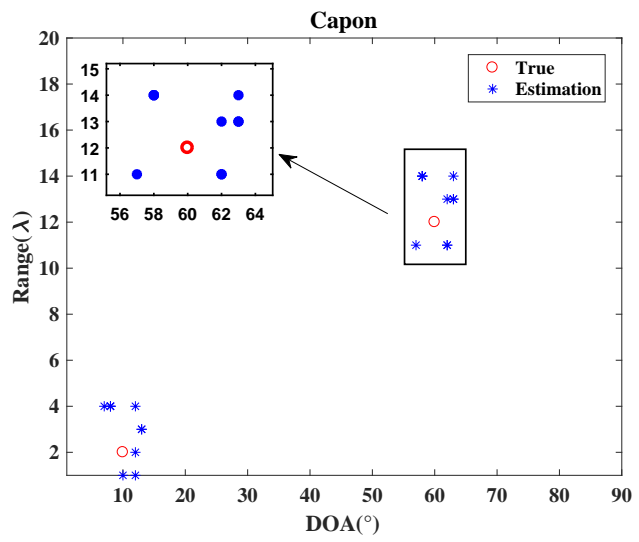

(c)

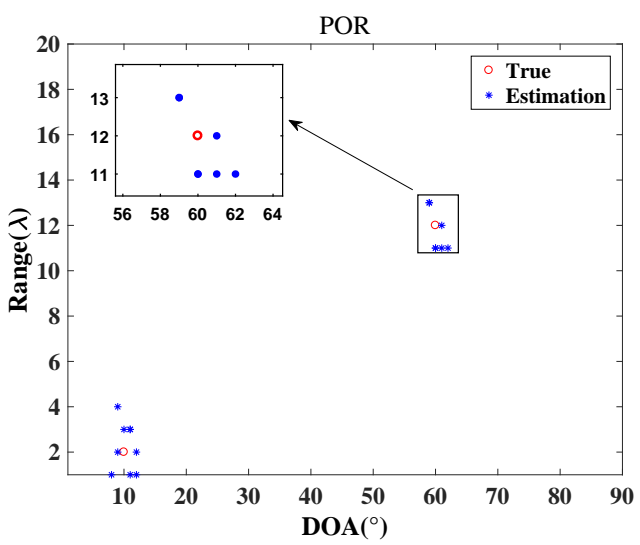

(b)

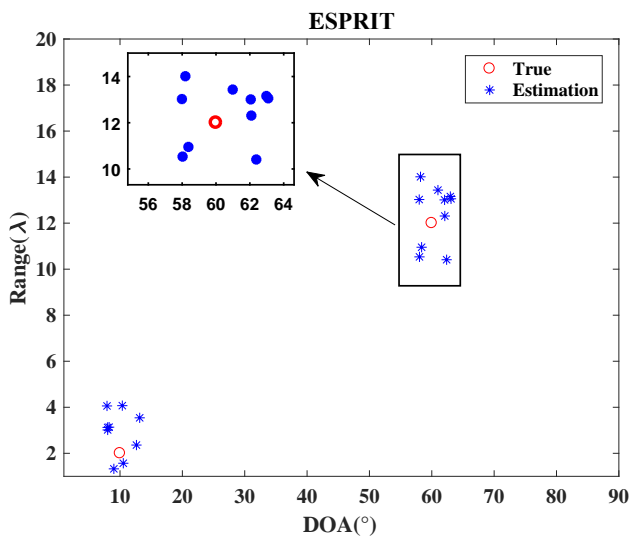

(d)

Figure 2: Near-field source localization with snapshots 100 and $\mathrm{SNR}=10 \mathrm{~dB}$. (a) MUSIC, (b) POR, (c) Capon, (d) ESPRIT. 


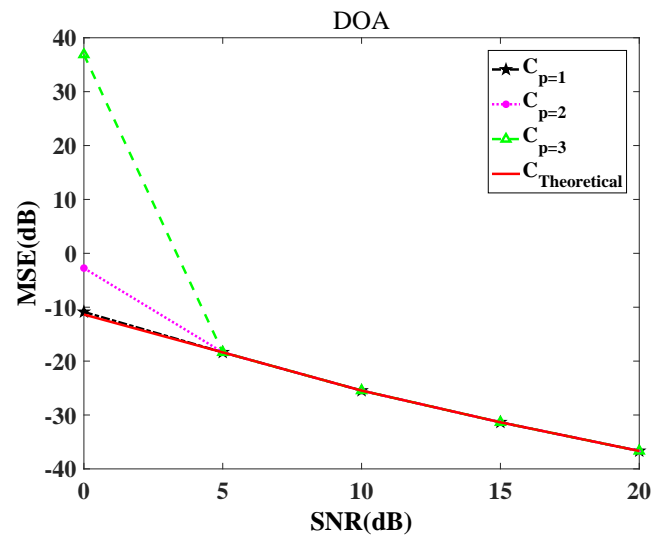

(a)

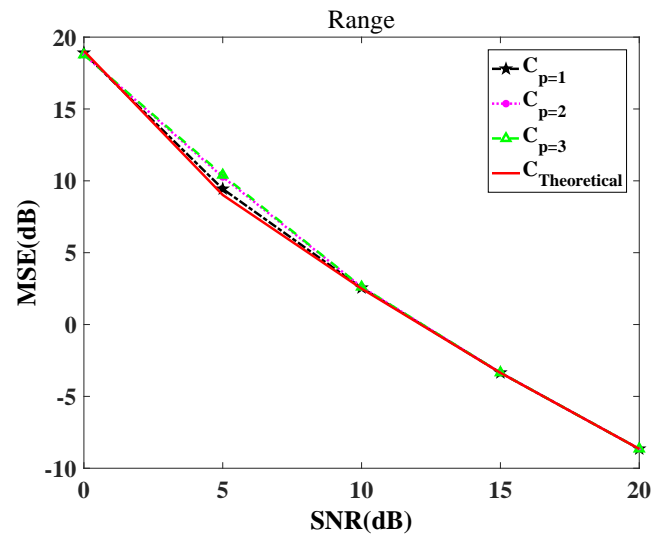

(c)

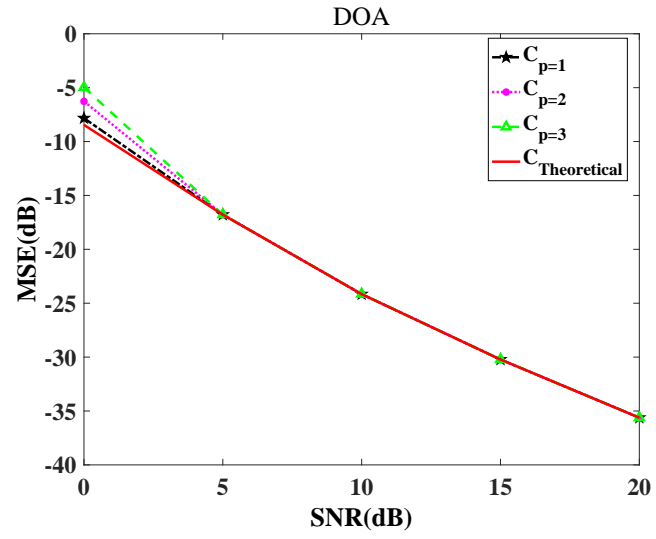

(b)

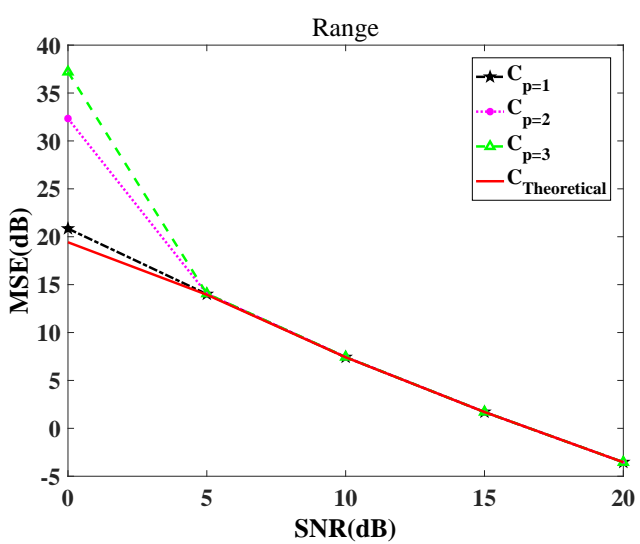

(d)

Figure 3: Performance evaluations of the POR method versus SNR. (a) DOA with full-bit measurement, (b) DOA with one-bit measurement, (c) Range with full-bit measurement, (d) Range with one-bit measurement. 
approach is faster than the Grid matching

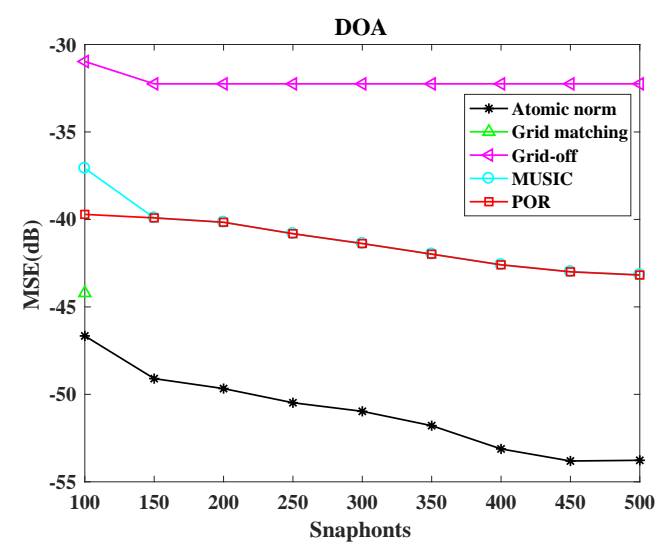

(a)

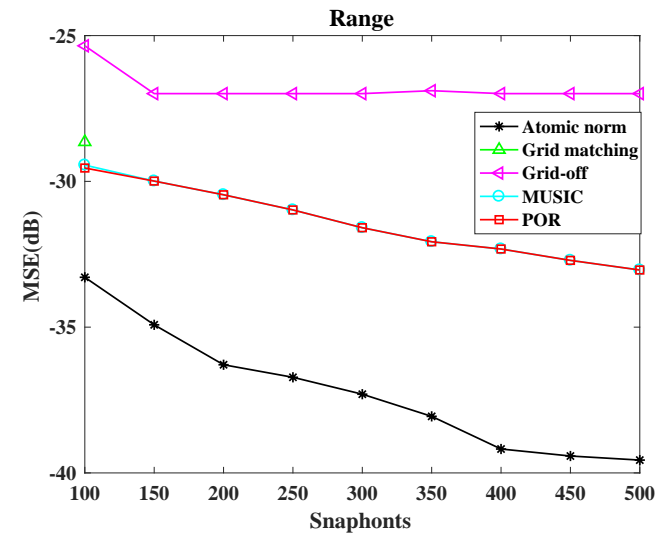

(c)

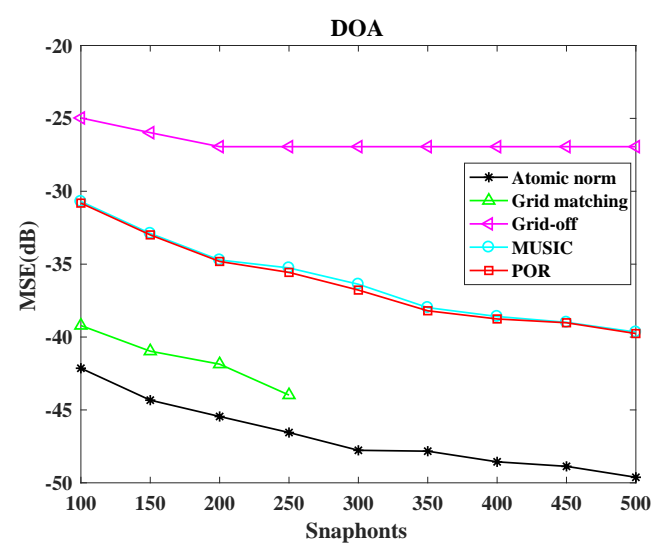

(b)

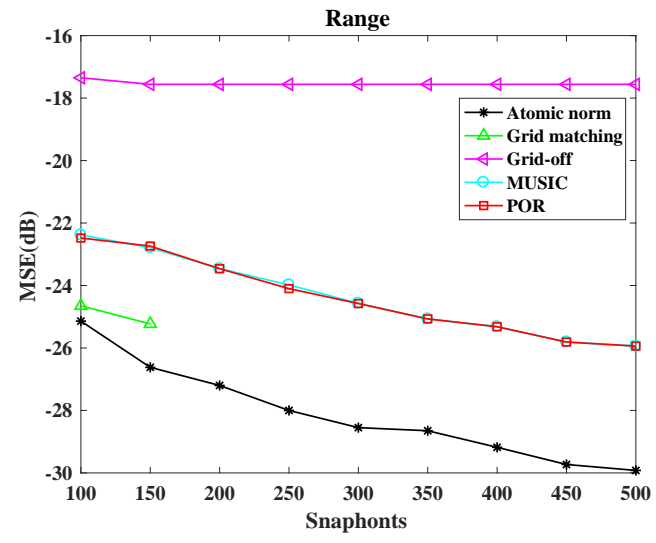

(d)

Figure 4: MSEs of different methods versus snapshots. (a) MSEs of DOA with full-bit, (b) MSEs of DOA with one-bit, (c) MSEs of range with full-bit, (d) MSEs of range with one-bit.

\subsection{Real-world data}

The real data are collected by a microphone array that is custom-made in our laboratory, where the array consists of five microphones and it is mounted at the bottom of a display and the spacing of sensors is $7 \mathrm{~cm}$. The data acquisition layout is depicted in Figure 6. A single tone signal with frequency 


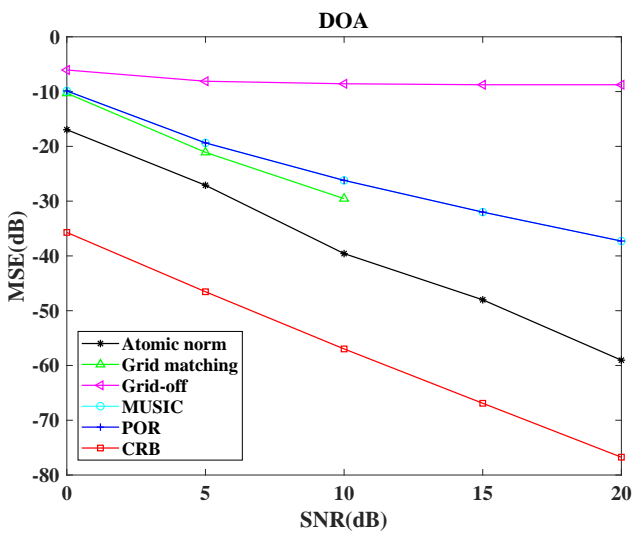

(a)

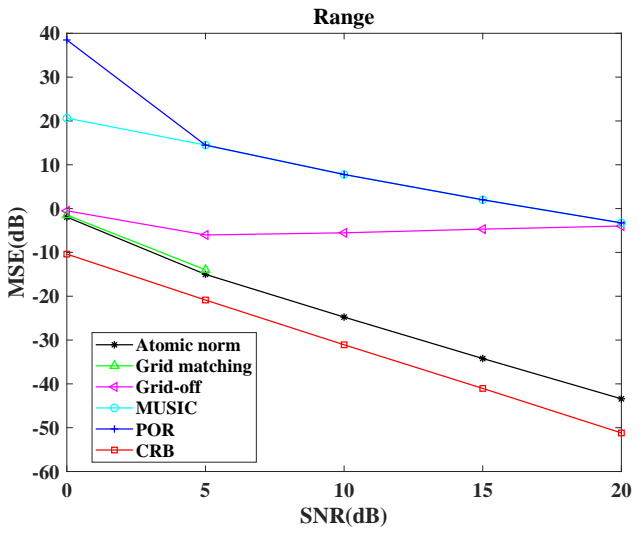

(c)

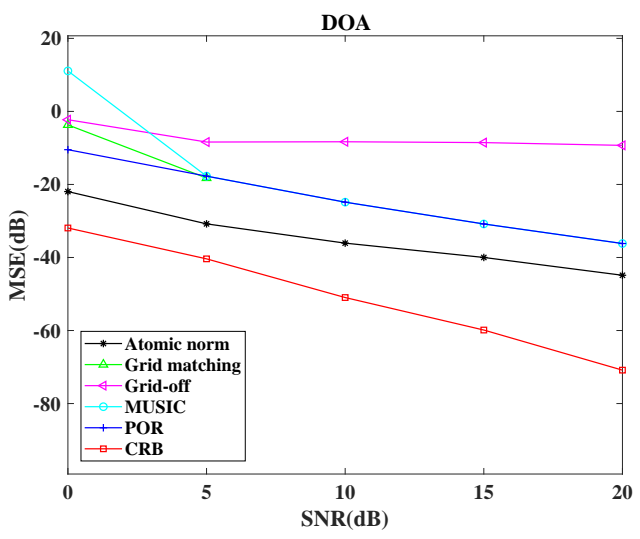

(b)

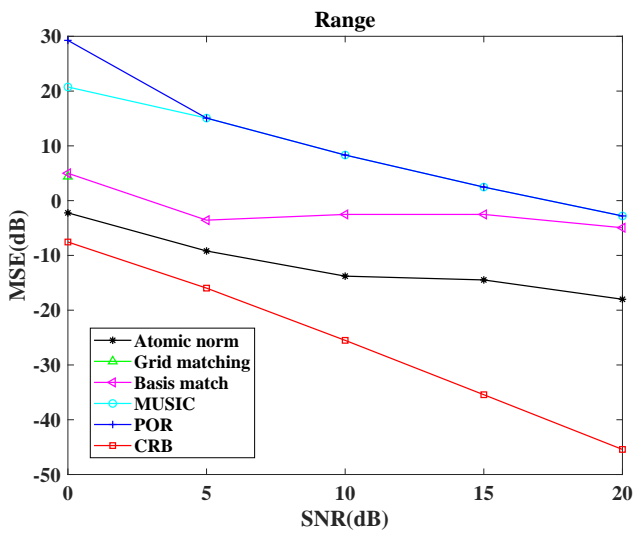

(d)

Figure 5: MSEs of different methods versus SNRs. (a) MSEs of DOA with full-bit, (b) MSEs of DOA with one-bit, (c) MSEs of range with full-bit, (d) MSEs of range with one-bit.

Table 2: Running times of different methods versus number of sensors (seconds)

\begin{tabular}{c|c|c|c|c}
\hline M & 3 & 5 & 7 & 9 \\
\hline MUSIC & 1.012 & 1.536 & 2.563 & 4.807 \\
\hline POR & 1.012 & 1.636 & 2.722 & 4.907 \\
\hline Grid matching & 3.917 & 5.773 & 7.088 & 7.296 \\
\hline Atomic norm & 1.503 & 1.761 & 2.153 & 2.721 \\
\hline
\end{tabular}


$1 \mathrm{kHz}$ is placed at $\left(30^{\circ}, 0.68 \mathrm{~m}\right)$ to act a single source and recording duration is 10 seconds. Another single tone signal with frequency $2 \mathrm{kHz}$ is placed at ${ }_{245}\left(60^{\circ}, 0.85 \mathrm{~m}\right)$ to act another single source and recording duration is 10 seconds. Finally, two single tone signals play simultaneously to generate multiple sources and the recording duration is also 10 seconds. From a simple calculation, the wavelengths of two sources are $\lambda_{1}=0.34 \mathrm{~m}$ and $\lambda_{2}=0.17 \mathrm{~m}$, which means the source positions are $\left(30^{\circ}, 2 \lambda_{1}\right)$ and $\left(60^{\circ}, 5 \lambda_{2}\right)$. The estimated results obtained by different approaches are provided in Figure 7. It is observed that all approaches are able to produce the estimated positions that match the true one well. In what follows, the case of broadband near-field is studied. A $16 \mathrm{kHz}$ sampled single speech signal is placed at $\left(30^{\circ}, 0.68 \mathrm{~m}\right)$ for a recording duration of 7 seconds, and another $16 \mathrm{kHz}$ sampled single speech signal is placed at $\left(60^{\circ}, 0.85 \mathrm{~m}\right)$ with a recording time of 7 seconds. Finally, the mixed speech signals of the those two are also collected with the same duration is 7 seconds. In the experiment, the minimum wavelength of the two speech signals is given by $\lambda_{3}=340 / 8000$, which means the source positions are at $\left(30^{\circ}, 16 \lambda_{3}\right)$ and $\left(60^{\circ}, 20 \lambda_{3}\right)$ by a simple calculation. The broadband near field estimation results obtained by different methods are provided in the Figure 8, where the positions are displayed in terms of $\lambda_{3}$. Obviously, all methods are also able to obtain the positions of sources in broadband near-field source location. However, the POR approach does not require knowing the number of sources and EVD, which is more practical in real applications, whereas the atomic norm based approach does not need to construct the grided dictionary, which eliminates the off-grid issue.

\section{Conclusion}

To perform near-field source localization, in this work, based on subspace theory and sparse signal reconstruction theory, two approaches are devised to obtain DOA and range information in a two-stage manner. To that end, by a use of symmetry property of the ULA, two special sequences are constructed and each sequence only depends on one unknown parameter. Because of this 


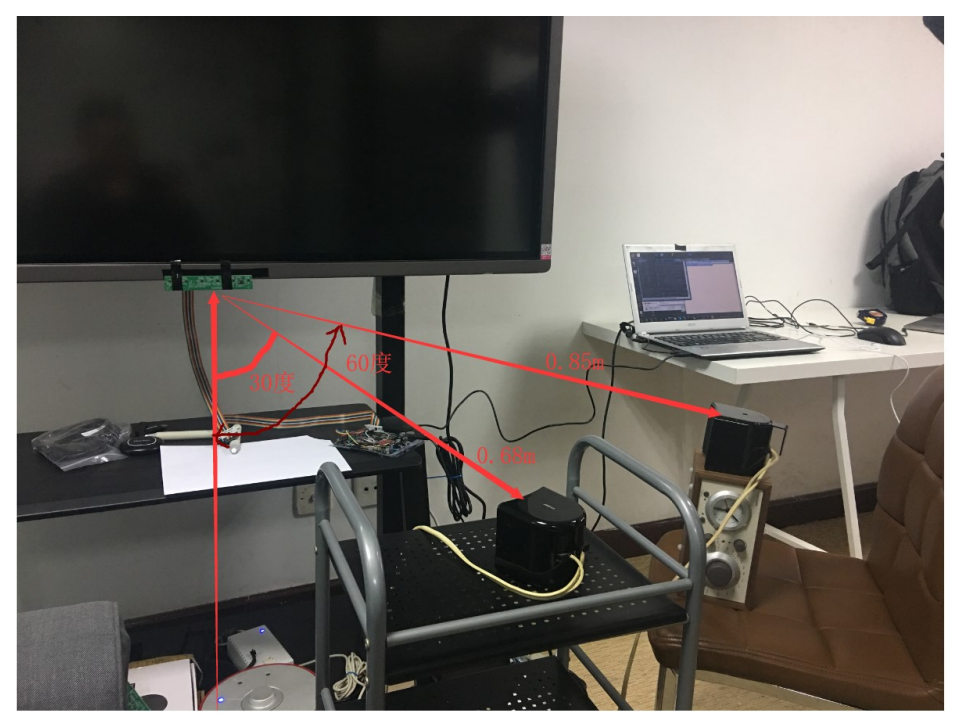

Figure 6: Experimental setup.

decouple operation, the computational complexity is obviously reduced since the search only confines to one parameter. In addition to the usual noise effect experiments, the proposed method is also examined under the case of one-bit quantization. The numerical studies including simulation and real-world data are provided to demonstrate that the proposed approach is able to localize the sources when they are in near-field.

\section{References}

[1] H. L. V. Trees, Optimum Array Processing: Part IV of Detection, Estimation, and Modulation Theory, John Wiley \& Sons, Inc., 2002.

[2] S. Makino, Audio Source Separation, Springer, 2018.

[3] J. Benesty, J. Chen, Y. Huang, Microphone Array Signal Processing, Springer, 2008.

[4] P. Stoica, A. Nehorai, MUSIC, maximum likelihood, and Cramer-Rao bound, IEEE Trans. Acoustics, Speech and Signal Processing 37 (5) (1989) $720-741$. 


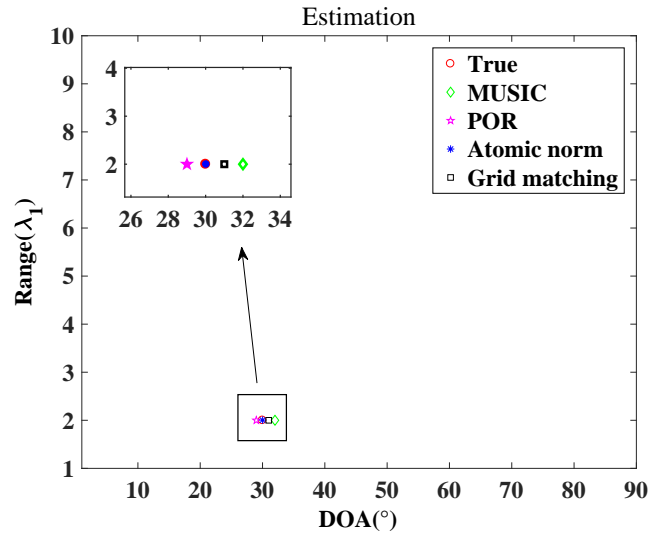

(a)

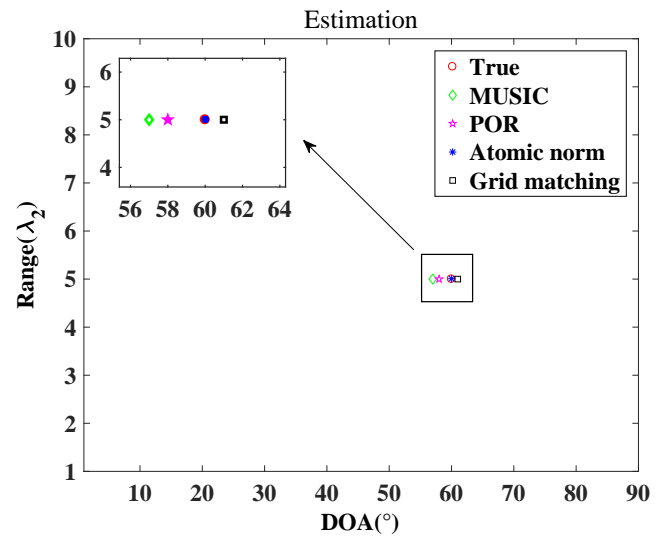

(b)

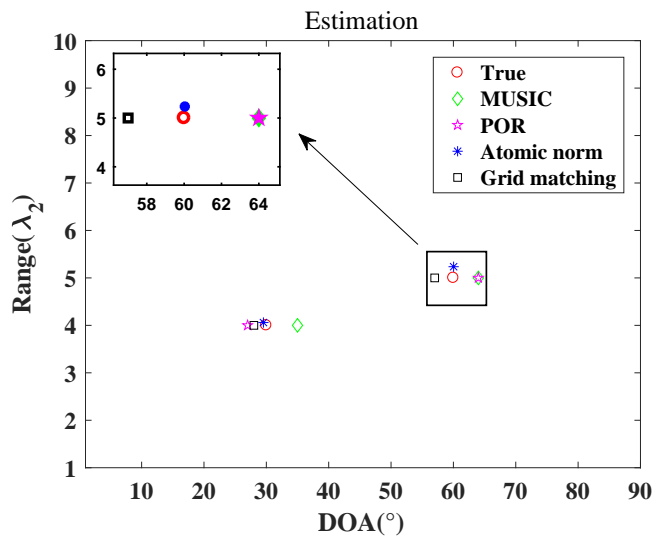

(c)

Figure 7: Real data estimation results for different methods of narrow-band near-field sources. (a) Localization of first source, (b) Localization of second source, (c) Localization of multiple sources. 


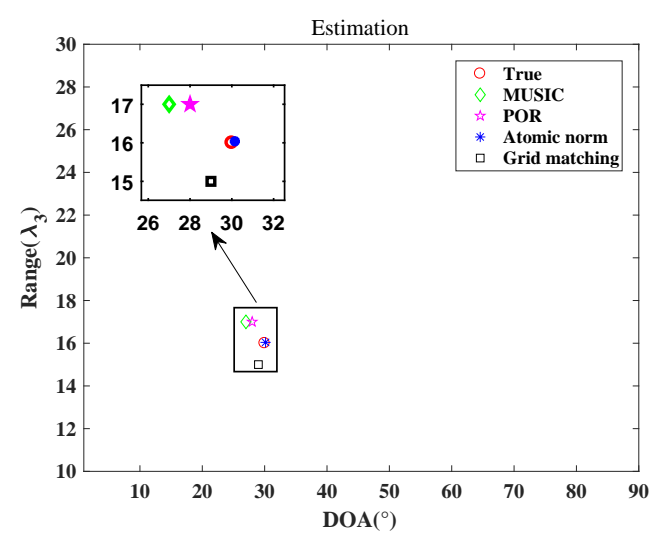

(a)

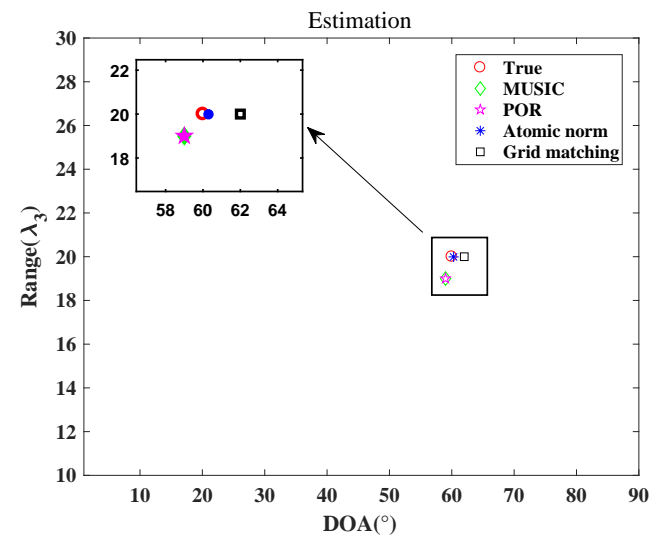

(b)

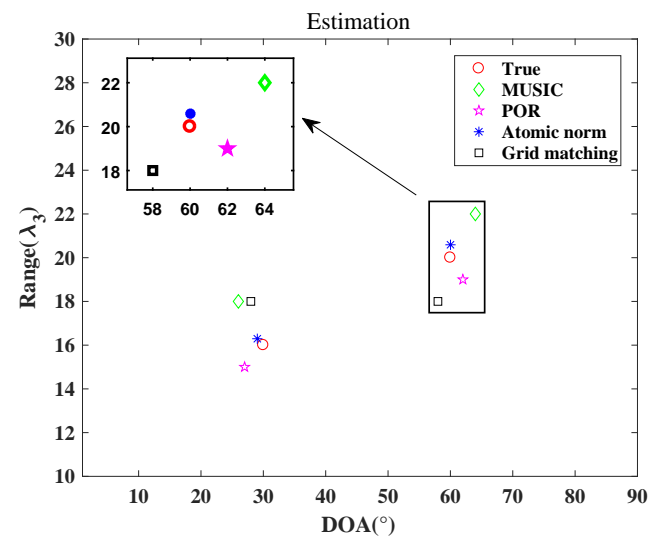

(c)

Figure 8: Real data estimation results for different methods of broadband nearfield sources.(a) Localization of first source, (b) Localization of second source, (c) Localization of multiple sources. 
[5] A. G. Jaffer, Maximum likelihood direction finding of stochastic sources: A separable solution, Proc. ICASSP 5 (1988) 2893-2896.

[6] A. Swindlehurst, M. Viberg, Subspace fitting with diversely polarized antenna arrays, IEEE Trans. Antennas Propagation 41 (1) (1993) 1687-1694.

[7] A. Paulraj, R. Roy, T. Kailath, A subspace rotation approach to signal parameter estimation, IEEE Trans. Signal Processing 74 (1986) 1044-1046.

[8] R. Kumaresan, D. W. Tufts, Estimating the angles of arrival of multiple plane waves, IEEE Trans. Aerosp. Electron. Syst. 19 (1) (1983) 134-139.

295 [9] D. Malioutov, M. Cetin, A. S.Willsky, A sparse signal reconstruction perspective for source localization with sensor arrays, IEEE Trans. Signal Processing 53 (8) (2005) 3010-3022.

[10] Z. M. Liu, Z. T. Huang, Y. Y. Zhou, Direction-of-arrival estimation of wideband signals via covariance matrix sparse representation, IEEE Trans. Signal Processing 59 (9) (2011) 4256-4270.

[11] P. Stoica, P. Babu, J. Li, SPICE: A sparse covariance-based estimation method for array processing, IEEE Trans. Signal Processing 59 (2) (2011) $629-638$.

[12] G. Tang, B. N. Bhaskar, P. Shah, B. Recht, Compressed sensing off the grid, IEEE Trans. Information Thorey 59 (11) (2013) 7465-7490.

[13] B. N. Bhaskar, G. Tang, B. Recht, Atomic norm denoising with applications to line spectral estimation, IEEE Trans. Singal Processing 61 (12) (2013) 5987-5999.

[14] Z. Yang, L. Xie, On gridless sparse methods for line spectral estimation from complete and incomplete data, IEEE Trans. Singal Processing 63 (12) (2015) 3139-3153.

[15] Z. Yang, L. Xie, Enhancing sparsity and resolution via reweighted atomic norm minimization, IEEE Trans. Singal Processing 64 (4) (2016) 995-1006. 
[16] Y. Zhou, C. Huang, H. Liu, D. Li, T.-K. Truong, Clutter removal in through-the-wall radar based on weighted nuclear norm minimization, IEEE Geoscience and Remote Sensing Letters, IEEE Early Access.

[17] K. Hu, S. Chepuri, G. Leus, Near-field source localization: Sparse recovery techniques and grid matching, IEEE 8th Sensor Array and Multichannel Signal Processing Workshop (SAM), 2014.

[18] Y. D. Huang, M. Barkat, Near-field multipe source localization by passive sensor array, IEEE Trans. Antennas Propag. 39 (7) (1991) 968-975.

[19] M. Haardt, R. N. Challa, S. Shamsunder, Improved bearing and range estimation via high-order subspace based unitary ESPRIT, Proc. 30th Asilomar Conf. Signals, Systems, and Computers, Pacific Grove, CA, 1996.

[20] J. C. Chen, R. E. Hudson, K. Yao, Maximum-likelihood source localization and unknown sensor location estimation for wideband signals in the nearfield, IEEE Trans. Signal Process. 50 (8) (2002) 1843-1854.

[21] E. Grosicki, K. Abed-Meraim, Y. Hua, A weighted linear prediction method for near-field source localization, IEEE Trans. Signal Processing 53 (10) (2005) 3651-3660.

[22] G. Liu, X. Sun, Efficient method of passive localization for mixed far-field and near-field sources, IEEE Antennas and Wireless Propagation Letters 12 (2013) 902-905.

[23] B. Wang, Y. Zhao, J. Liu, Mixed-order MUSIC algorithm for localization of far-field and near-field sources, IEEE Signal Processing Letters 20 (4) (2013) 311-314.

[24] W. Zuo, J. Xin, N. Zheng, A. Sano, Subspace-based localization of far-field and near-field signals without eigendecomposition, IEEE Signal Processing Letters 66 (17) (2018) 4461-4476. 
[25] O. Bar-Shalom, A. J. Weiss, DOA estimation using one-bit quantized measurements, IEEE Trans. Aerosapce and Electronic Systems 38 (3) (2002) 868-884.

[26] Y. Gao, D. Hu, Y. Chen, Y. Ma, DOA estimation from one-bit compressed array data via joint sparse representation, IEEE Signal Processing Letters 23 (9) (2016) 1279-1283.

[27] Y. Gao, D. Hu, Y. Chen, Y. Ma, Gridless 1-b DOA estimation exploiting SVM approach, IEEE Communications Letters 21 (10) (2017) 868-884.

[28] J. W. Goodman, Introduction to Fourier optics, New York: McGraw-Hill, 1996.

[29] Y. Wu, H. C. So, H. Q. Liu, Subspace-based algorithm for parameter estimation of polynomial phase signals, IEEE Trans. Signal Processing 56 (10) (2008) 4977-4983.

[30] Z. Xu, P. Liu, X. Wang, Blind multiuser detection: From MOE to subspace methods, IEEE Trans. Signal Processing 52 (2) (2004) 510-524.

[31] H. Q. Liu, G. S. Liao, Y. Xie, J. Zhang, Unified framework for two robust beamforming methods, Electronics Letters 42 (7) (2006) 425-426.

[32] T. H. Liu, J. M. Mendel, Azimuth and elevation direction finding using arbitrary array geometries, IEEE Trans. Signal Processing 46 (7) (1998) 2061-2065.

[33] D. Chen, B. Chen, G. Qin, Angle estimation using ESPRIT in MIMO radar, Electronics Letters 44 (12) (2008) 770-771.

[34] H. Q. Liu, D. Li, Y. Zhou, T.-K. Truong, Simultaneous radio frequency and wideband interference suppression in SAR signals via sparsity exploitation in time-frequency domain, IEEE Trans. Geoscience and Remote Sensing 56 (10) (2018) 5780-5793. 
[35] P. Zhang, L. Gan, C. Ling, S. Sun, Atomic norm denoising-based joint channel estimation and faulty antenna detection for massive MIMO, IEEE Trans. Vehicular Technology 67 (2) (2018) 1389-1403.

[36] M. Grant, S. Boyd, Y. Ye, CVX: Matlab software for disciplined convex programming, [Online]. Available: http://www.stanford.edu/ boyd/cvx/.

[37] J. H. V. Vleck, D. Middleton, The spectrum of clipped noise, Proceedings of the IEEE 54 (1) (1966) 2-19.

[38] G. Jacovitti, A. Neri, Estimation of the autocorrelation function of complex Gaussian stationary process by amplitude clipped signals, IEEE Trans. Information Theory 40 (1) (1994) 239-245. 